\title{
Post-traumatic Anterior Cerebral Artery Rupture After a Severe Traumatic Brain Injury
}

\author{
${ }^{1}$ Quentin Mathais, ${ }^{2}$ Pierre Esnault, ${ }^{3}$ Christophe Joubert, ${ }^{4}$ Caroline Dragone, ${ }^{5}$ Eric Meaudre
}

\begin{abstract}
Blunt cerebrovascular injuries (BCVI) have been increasingly recognized in the past decade due to the initiation of different screening protocols. We present the case of an anterior cerebral artery rupture with free contrast extravasation following a severe traumatic brain injury. Epidemiology, modalities of screening and treatment of $\mathrm{BCVI}$ are discussed. This report reminds that the screening of BCVI may be essential after a severe TBI.
\end{abstract}

Keywords: Anterior cerebral artery, Blunt cerebrovascular injury, Traumatic brain injury.

How to cite this article: Mathais $Q$, Esnault $P$, Joubert $C$, Dragone C, Meaudre E. Post-traumatic Anterior Cerebral Artery Rupture After a Severe Traumatic Brain Injury. Indian Journal of Critical Care Medicine, January 2019;23(1),54-55.

Source of support: Nil

\section{Conflict of interest: None}

Blunt cerebrovascular injuries (BCVI) have been increasingly recognized in the past decade due to the initiation of different screening protocols. ${ }^{1}$ We recently demonstrated that BCVI is frequent after severe traumatic brain injuries (TBI), with a prevalence up to $9 \% .^{2}$

A 57-year-old woman was admitted to the trauma center for severe TBI after she jumped out of a window. Initial physical examination revealed a Glasgow Coma scale score at 3, and her pupils were dilated and nonreactive. She also presented with hemorrhagic shock with diffuse facial bleeding. She was quickly stabilized after transfusion and infusion of norepinephrine. The wholebody CT scan showed depressed skull fractures, bilateral subdural hemorrhage, intraventricular hemorrhage, diffuse subarachnoid hemorrhage, right frontal contusion, and diffuse brain swelling (Fig. 1). The CT angiography showed a right anterior cerebral artery rupture with blush next to the frontal contusion (Fig. 2). An aneurysm was

\footnotetext{
${ }^{1-5}$ Researchers

${ }^{1,2,4}$ Intensive Care Unit, Sainte Anne Military Hospital, Toulon, France

${ }^{3}$ Departement of Neurosurgery, Sainte Anne Military Hospital, Toulon, France

${ }^{5}$ Intensive Care Unit, Sainte Anne Military Hospital, Toulon, France and French Military Health Service Academy Unit, Ecole du Val-de-Grâce, Paris, France

Corresponding author: Quentin Mathais, Researchers, Intensive Care Unit, Sainte Anne Military Hospital, Toulon, France, Phone: +33 483162 205, e-mail: quentin.mathais@orange.fr
}

not present. There was no other vessel injured. There were also multiples facial fractures. Given the severity of the injuries, neurosurgeons did not perform an emergent surgical decompression. Despite advanced medical care, the patient developed refractory intracranial hypertension leading to brain death the fourth day after the admission.

BCVI are not uncommon after blunt trauma. Recent studies found an incidence of approximately 1 to $2 \%{ }^{1}$ Severe TBI is a well-known risk factor of BCVI, with an incidence up to 5 -fold higher in this specific population. ${ }^{2}$ However, catching active bleeding due to an intracranial arterial injury is exceptional. BCVI occurs mainly in large extracranial vessels like carotid and vertebral arteries. ${ }^{1}$ Moreover, most of these cases concern low-grade injuries according to Denver scale. The Denver scale (or Biffl scale) is the most commonly used grading scheme in the literature and has been reused in the 2010 guidelines of the Eastern Association for the Surgery of Trauma to guide treatment (Table 1). ${ }^{1}$ In severe TBI, high-grade injuries (grades IV and V), as in the present report, represent only approximately $20 \%$ of the cases. ${ }^{2}$

Secondly, this case emphasizes the crucial importance of early detection of BCVI in TBI patients because of its therapeutic impact. Indeed, BCVI is potentially devastating because they can lead to neurovascular events. Untreated BCVIs of the carotid an vertebral arteries could have an overall stroke rate approaching 40 to $60 \%$, and a stroke-related mortality rate as high as $50 \% .{ }^{2,3}$ Recent studies showed that medical therapy with anticoagulation or antiplatelet agents that is started before the onset of neurological symptoms could reduce the occurrence of neurological events by a factor of 6 , decreasing the stroke rate as low as $4 \%$ in grade I to III patients. ${ }^{4}$ Some

Table 1: Denver grading of BCVI

\begin{tabular}{cl}
\hline $\begin{array}{c}\text { Grade I } \\
\text { Grade II }\end{array}$ & $\begin{array}{l}\text { Intimal irregularity with <25\% narrowing } \\
\text { Dissection or intramural hematoma with } \\
>25 \% \text { narrowing }\end{array}$ \\
Grade III & $\begin{array}{l}\text { Pseudoaneurysm } \\
\text { Grade IV }\end{array}$ \\
Occlusion \\
Grade V & Transection with extravasation \\
\hline Source: Bromberg WJ, Collier BC, Diebel LN, Dwyer KM, Holevar \\
MR, Jacobs DG, et al. Blunt cerebrovascular injury practice \\
management guidelines: the Eastern Association for the Surgery of \\
Trauma. J Trauma. 2010;68:587-95)
\end{tabular}



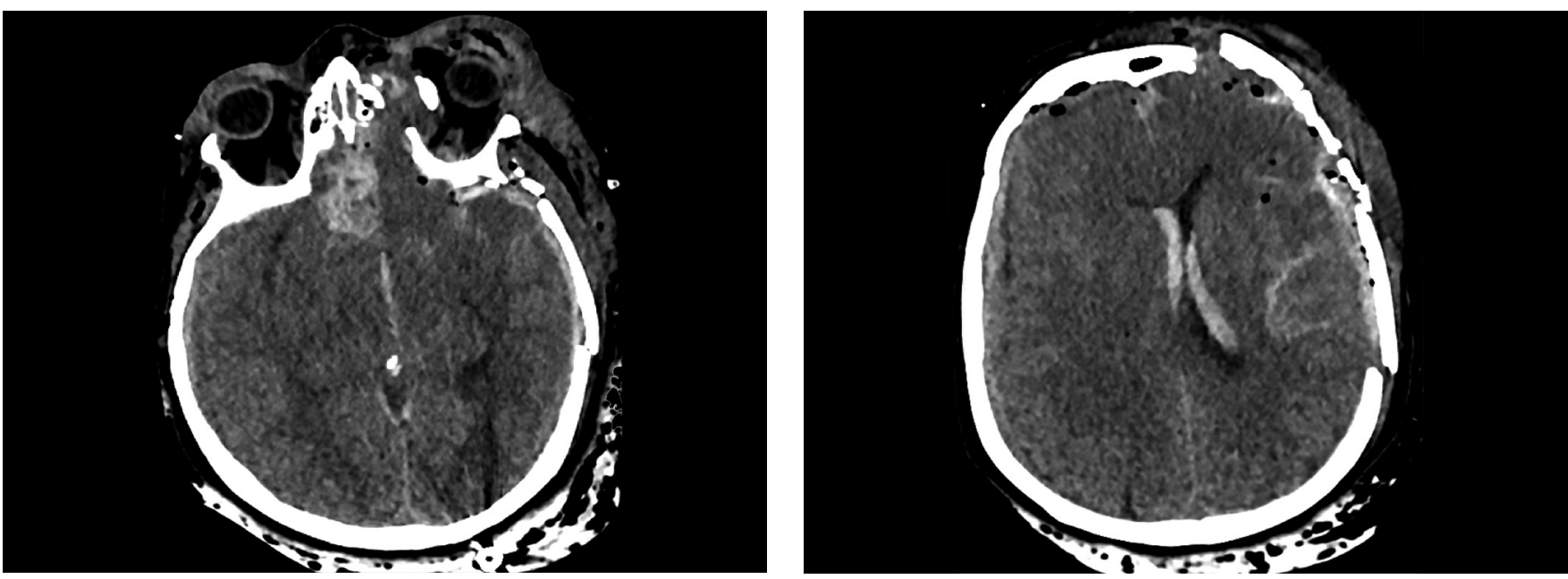

Figs 1A and B: (A) Axial head CT scanner without contrast showing the right frontal lobe contusion; (B) Axial head CT scanner without contrast showing bilateral subdural hemorrhage, intraventricular hemorrhage, diffuse subarachnoid hemorrhage, pneumocephalus associated with depression of the frontal and parietal bones with fracture

practitioners could have a doubt concerning the safety of this therapy in patients with severe TBI. However, studies including individuals with neurological injury (TBI or spinal cord injury) did not show an increased risk of intracranial hemorrhage progression after early initiation of pharmacological treatment. ${ }^{2,3}$ In the case of high-grade injuries (grades IV and V), endovascular interventions with coiling could be of interest.

The modalities of BCVI screening evolved greatly over the past 2 decades. Digital four vessel subtraction angiography has been considered the standard imaging modality. ${ }^{1}$ Nowadays, CT angiography using 32-channel and higher multidetector CT scanners has supplanted subtraction angiography as a first-line screening modality, with a sensitivity of 66 to $98 \%$, a specificity of 92 to $100 \%$ and a good cost-efficiency ratio. ${ }^{5} \mathrm{MR}$ imaging is currently not considered an appropriate tool for BCVI screening. However, it allows a good vessel wall imaging and may be considered for follow up BCVI evaluation. ${ }^{5}$

To our knowledge, this is the first report of a complete anterior cerebral artery transection with free contrast extravasation. This report emphasizes that screening of BCVI may be essential after a severe TBI if there is a suspicion of vascular injury on the initial noncontrast CT scan, and even more with the widespread availability of high-performance CT scanners which permits a fast, efficient and low-cost examination of vascular structures.

\section{Abbreviations}

BCVI: Blunt cerebrovascular injury

TBI: Traumatic brain injury

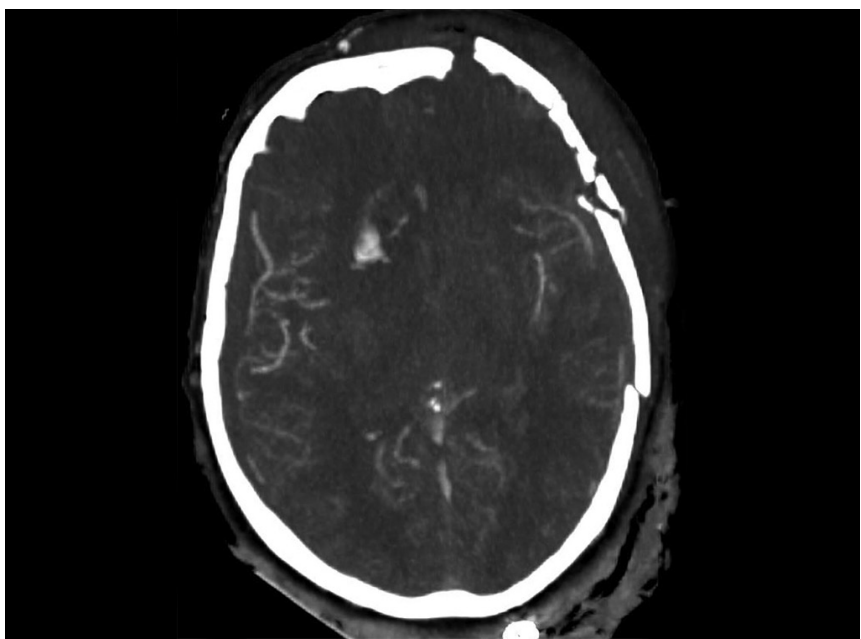

Fig. 2: Axial head CT scanner with angiography showing a right anterior cerebral artery rupture with free contrast extravasation.

\section{REFERENCES}

1. Bromberg WJ, Collier BC, Diebel LN, Dwyer KM, Holevar MR, Jacobs DG, et al. Blunt cerebrovascular injury practice management guidelines: the Eastern Association for the Surgery of Trauma. J Trauma. 2010;68:587-95.

2. Esnault $\mathrm{P}$, Cardinale $\mathrm{M}$, Boret $\mathrm{H}, \mathrm{D}^{\prime}$ Aranda $\mathrm{E}$, Montcriol A, Bordes J, et al: Blunt cerebrovascular injuries in severe traumatic brain injury: incidence, risk factors, and evolution. J Neurosurg. 2017;127(1):16-22.

3. Callcut RA, Hanseman DJ, Solan PD, Kadon KS, Ingalls NK, Fortuna GR, et al: Early treatment of blunt cerebrovascular injury with concomitant hemorrhagic neurologic injury is safe and effective. J Trauma Acute Care Surg. 2012;72:338-46.

4. Markus HS, Hayter E, Levi C, Feldman A, Venables G, Norris $\mathrm{J}$ : Antiplatelet treatment compared with anticoagulation treatment for cervical artery dissection (CADISS): a randomized trial. Lancet Neurol. 2015;14:361-7.

5. Rutman AM, Vranic JE, Mossa-Basha M: Imaging and Management of Blunt Cerebrovascular Injury. Radiographics. 2018;38(2):542-63. 